\title{
Criminologie
}

\section{Théories et pratiques criminologiques : quand l'organisation mène le jeu}

\section{Guy Lemire}

Volume 19, numéro 1, 1986

Politiques et pratiques pénales. 25 ans de réflexion et d'action

URI : https://id.erudit.org/iderudit/017233ar

DOI : https://doi.org/10.7202/017233ar

Aller au sommaire du numéro

Éditeur(s)

Les Presses de l'Université de Montréal

ISSN

0316-0041 (imprimé)

1492-1367 (numérique)

Découvrir la revue

Citer cet article

Lemire, G. (1986). Théories et pratiques criminologiques : quand l'organisation mène le jeu. Criminologie, 19(1), 215-226. https://doi.org/10.7202/017233ar

\section{Résumé de l'article}

The analysis of plea bargaining and diversion shows how criminological theories and principles are shaped by organizational constraints; it also indicates the limits of theories and the need for practical knowledge. The concepts of control and power which are so suspicious to criminologists, should be replaced in an organizational perspective in order to give a new meaning to knowledge and praxis. 


\section{THÉORIES ET PRATIQUES CRIMINOLOGIQUES : QUAND L'ORGANISATION MÈNE LE JEU}

Guy Lemire*

The analysis of plea bargaining and diversion shows how criminological theories and principles are shaped by organizational constraints; it also indicates the limits of theories and the need for practical knowledge. The concepts of control and power which are so suspicious to criminologists, should be replaced in an organizational perspective in order to give a new meaning to knowledge and praxis.

Une science économique coupée de la problématique du pouvoir est dépourvue de signification et pertinence

J.K. Galbraith

«Notre société est une société de l'organisation», écrit Etzioni ${ }^{1}$. Galbraith ${ }^{2}$ parle de el'essor de l'organisation». Ces énoncés, en apparence anodins, résument une vérité lourde de conséquences : les théories, principes et politiques cèdent le pas des impératifs organisationnels de plus en plus envahissants. On peut presque parler de prise de possession et/ou de récupération. Nous voulons done dans cet article mesurer tout d'abord le sort fait par l'organisation à nos grands principes de justice et à nos mesures les plus généreuses. Il nous sera par la suite possible d'en évaluer les conséquences.

\section{$-1-$}

Il n'est pas de principe plus fondamental dans notre système de justice que la présomption d'innocence. Tout accusé est présumé innocent jusqu'à preuve du contraire. Voilà bien un principe qui fait la fierté d'une société démocratique. Or, que devient ce prin-

- Guy Lemire, professeur agrégé, École de criminologie, Université de Montréal.

1. Etzioni, A. (1971), les Organisations modernes, Gembloux, Duculot. Sevil.

2. Galbraith, J.K. (1985), Anatomie du pouvoir, Paris, Éditions du 
cipe dans la réalité? Littrell ${ }^{3}$ a analysé le fonctionnement des cours de justice et a découvert que $90 \%$ des causes criminelles se règlent par voie de négociation de culpabilité. Pour lui, le fonctionnement des tribunaux est, dans les faits, organisé selon une présomption de culpabilité et non plus d'innocence. Dans la très grande majorité des cas, il est clair que le principe fondamental de notre système de justice n'est pas retenu : il est même bafoué, et ce, au su et au vu de tout le monde. Pourquoi en est-on rendu là et pourquoi, finalement, s'en indigne-t-on si peu? Parce que les exigences organisationnelles l'emportent sur les principes les plus importants. On se plaint déjà de l'encombrement des cours de justice et des longs délais. Dans ces conditions, peut-on même imaginer la situation s'il fallait qu'un procès ait lieu dans chaque cas. Ce serait évidemment catastrophique, notamment sur le plan financier. Pour Littrell, le constat est clair : la bureaucratie a modifié le processus judiciaire. Des normes maintenant supérieures aux règles de droit se sont imposées et ce, dans le sanctuaire même du droit pénal.

Littrell a également le mérite de démontrer que ce déplacement de valeurs a amené un déplacement de pouvoir: les personnes clés du système judiciaire ne sont plus les juges mais les procureurs et les policiers : en d'autres mots, ceux autour de qui se joue la négociation de culpabilité et de sentence. Il en est d'ailleurs ainsi dans la plupart des grandes organisations formelles et bureaucratiques: les gens les plus importants se retrouvent dans les échelons intermédiaires, là où «s'opérationnalisent» les politiques et procédures. Dans le système judiciaire, les juges, dans la très grande majorité des situations, ne font qu'officialiser ce qui a été décidé à des échelons «inférieurs». En outre, les ingrédients essentiels de la négociation sont bien connus : l'importance des crimes commis, les antécédents et la nécessité de punition.

Il reste une question fondamentale : puisqu'il en est ainsi, justice est-elle moins bien rendue? Avant de répondre directement à la question, il faut se demander si les normes légales sont aussi essentielles qu'on le prétend. Le droit, c'est l'avènement du formalisme, de la procédure. Or, l'analyse des mécanismes formels nous a déjà enseigné que ceux-ci se préoccupent plus de la forme que du fond, plus d'abstraction que de réalité, plus des choses que des

3. Littrell, W.B. (1979), Bureaucratic /ustice, Beverly-Hills, Sage Library of Social Research. 
gens, plus des procédures que de la qualité. Dans les organisations, les modèles formels sont de plus en plus dépassés, «la vieille rationalité... a cessé d'être une discipline utile»". Il y a d'ailleurs de bonnes raisons de penser que, si les exigences organisationnelles ont eu raison des normes légales, c'est que finalement elles rendaient mieux compte de la réalité. La justice c'est beaucoup plus qu'un code, de la même façon que le contrôle et la réinsertion d'un ex-détenu c'est autre chose qu'un manuel de gestion de cas La vie réelle d'une organisation se situe bien au-delà des définitions officielles et ce sont les mécanismes non formels qui en assurent le fonctionnement essentiel.

Lorsqu'il s'agit de porter un jugement global sur le système judiciaire, Littrell en souligne tout d'abord les lacunes : il n'y a pas de véritables négociations et il est difficile de démontrer son innocence dans un modèle basé sur la présomption de culpabilité. En conclusion toutefois, Littrell fait sienne la conclusion de Silberman ${ }^{6}$ : "A seemingly irrational and unjust adult judicial process produces results that are surprisingly rational and just.» En d'autres termes, même s'il s'appuie sur des exigences officieuses tout à fait opposées aux définitions offioielles, on peut penser que le système judiciaire n'en fonctionne pas moins bien pour cela.

\section{$-2-$}

Parmi les idées les plus intéressantes développées par la criminologie, la déjudiciarisation figure en tête de liste. Compte tenu de l'échec et des limites des mesures traditionnelles, ce concept semblait devoir s'imposer comme une solution d'avenir. La Commission de réforme du droit ${ }^{7}$ en recommandait «la libéralisation et la légitimation» afin qu'elle constitue "dorénavant une option judicieuse dans les rouages de l'administration de la justice pénale». C'est dans le secteur des mineurs délinquants que cette nouvelle pratique a été le plus développée. Quels sont les résultats?

4. Peters, T., R. Waterman (1983), le Prix de l'excellence, Paris, Inter Editions.

5. Lemire, G. (1981), "La libération conditionnelle : le point de vue de la sociologie des organisations», in Criminologie, 1981, vol. 14, no 2 , Presses de l'Université de Montréal.

6. Silberman, C.E. (1978), Criminal Violence, Criminal Iustice, New York, Random House.

7. Commission de réforme du droit du Canada (1975), Études sur la déjudiciarisation, Ottawa, Information Canada. 
Doob $^{8}$ nous rappelle tout d'abord que «le tribunal pour mineurs fut à l'origine lui-même institué comme solution de rechange à une structure supposée plus formelle, à savoir le tribunal pour adultes». Aujourd'hui, ce sont les tribunaux de jeunes qui sont jugés trop formels. Or, comme le note Doob ${ }^{9}$, afin d'éviter une structure formelle (le tribunal pour mineurs) une autre structure formelle (au Québec, la D.P.J.) est mise sur pied. Le formalisme judiciaire a cédé la place au formalisme bureautcratique. Aussi, Doob ${ }^{10}$ est-il justifié d'ironiser sur «l'implantation d'une institution formelle, afin de permettre le traitement informel des jeunes contrevenants».

La question fondamentale mérite maintenant d'être posée : la déjudiciarisation a-t-elle eu comme effet de diminuer le nombre d'interventions judiciaires? Pour Doob ${ }^{11}$, le constat est clair : elle ne l'a pas fait. Elle s'est plutôt substituée au «tamisage effectué par la police» de telle sorte que «dans les programmes de déjudiciarisation conçus comme des solutions alternatives au passage au tribunal, on retrouve une proportion importante de jeunes qui de toute façon n'auraient pas été traduits devant le tribunal».

Dans un second article au titre révélateur ${ }^{12}$, Doob souligne que la formalisation de la déjudiciarisation a transformé les décisions des policiers en non-décisions où les policiers s'abstiennent de donner suite et réfèrent systématiquement les jeunes au D.P.J. Bel exemple d'une pratique courante, réalisée de façon non formelle. qui ne s'améliore guère quand on tente de l'organiser de façon plus systématique. Trépanier ${ }^{13}$ va même plus loin lorsqu'il conclut : "Il semble bien que l'extension du contrôle social soit un problème dont il faille accepter l'existence à partir du moment où on forma-

8. Doob, A.N. (1980), "Montée et déclin de la déjudiciarisation dans la législation sur les mineurs délinquants au Canada», Déviance et société, vol. $4, n^{0} 3$, p. $231-243$.

\section{Ibid.}

10. Ibid.

11. Ibid.

12. Doob, A.N. (1983), «Turning decisions into non-decisions», in R. Corrado, M. LeBlanc, et I. Trépanier (édit.), Current issues in iuvenile justice, Toronto, Butterworths.

13. Trépanier, J. (1984), «Mineurs en danger et mineurs délinquants : deux régimes en transformation au Québec et au Canada», Revue de droit pénal et de criminologie, novembre 1984, p. 817-848. 
lise des programmes de déjudiciarisation s'insérant entre la police et les tribunaux.»

Certains pourraient être tentés de penser qu'il s'agit d'un problème strictement relié à la Loi sur la protection de la jeunesse (Loi 24) mais Trëpanier ${ }^{14}$ ajoute : «Il n'y a aucune raison de croire que la nouvelle Loi sur les jeunes contrevenants ou les décrets la complétant puisse y changer quoi que ce soit». Par ailleurs, du côté de la délinquance adulte, si on considère les mesures qui ont comme objectif de réduire l'impact du système pénal sur les clientèles, le problème demeure le même. L'évaluation des sentences de travaux communautaires ${ }^{15}$, conçues comme alternatives aux courtes peines de prison, conclut que «ce sont avant tout les procédures qui éliminent de facto un nombre important de clients potentiels». De plus, «les modalités afférentes au processus de sélection des candidats... donnent lieu à un tel élagage de la population qu'il est permis de supposer que la clientèle finalement admise au programme en est une qui est plus que triée sur le volet et qui en regard de l'objectif fondamental du programme ne représente qu'une infime proportion de la clientèle potentielle visée par les sentences de travaux communautaires». Somme toute, il est loin d'être sûr que la clientèle touchée était bien la clientèle visée. De là à parler, encore ici, d'extension du contrôle social, il n'y a qu'un pas qui peut être vite franchi.

Nous aurons l'occasion de revenir plus tard sur cette notion d'extension du contrôle social mais ce que les dernières pages nous ont appris, c'est que les mesures les plus généreuses ont échoué pour des raisons qui n'ont probablement rien à voir avec la qualité intrinsèque de ces mesures mais qui touchent avant tout les choix organisationnels et les modalités opératoires.

Cette conclusion peut servir à la fois de mise en garde et de défi aux abolitionnistes et à tous ceux qui recherchent les mesures idéales. Peut-être concentrons-nous trop nos énergies au mauvais endroit : il est moins prioritaire d'identifier quoi faire que d'établir comment faire. Cette recherche de LA mesure ultime est-elle un progrès ou une course en circuit fermé où on refait continuellement

14. Trépanier, J., ibid.

15. Simon, H., S. Vallières (1981), l'Évaluation d'une nouvelle mesure pénale : les travaux communautaires au Québec, Montréal, École de criminologie. 
le même parcours? Le savoir-faire criminologique et organisationnel n'est-il pas plus important que l'idéologie, si séduisante soitelle. L'idéologie criminologique des années 60 est devenue pour plusieurs le cauchemar des années 80 . Pourquoi en serait-il autrement pour l'abolitionnisme quand le savoir-faire marque le pas? En criminologie, l'idéologie est-elle autre chose qu'une mode? Les abolitionnistes s'appuient sur un formalisme plus administratif que pénal qui n'a guère fait ses preuves à ce jour comme les études sur la déjudiciarisation l'ont démontré. L'abolitionnisme ne risque. t-il pas de devenir l'extension ultime du contrôle social?

Cette prépondérance de l'organisation a des conséquences qu'on n'a guère évaluées à ce jour. Tous constatent que la pratique criminologique se réalise dans des organisations de diverses natures et que ces organisations peuvent faciliter ou compliquer les choses. Toutefois, nous ne connaissons guère de recherches ou d'écrits qui ont tenté de mesurer les conséquences d'un tel état de choses sur le savoir et l'expertise. L'organisation a ses exigences propres lesquelles, comme on l'a vu, modifient considérablement nos principes et nos théories. Où cela risque-t-il de nous mener?

En premier lieu, il faut souligner combien le formalisme a peu d'emprise sur la réalité. Dans un premier cas, la présomption d'innocence, un principe fondamental du droit, est carrément bafouée; dans un deuxième cas, la déjudiciarisation, l'organisation formelle d'une telle mesure ne rejoint pas la clientèle visée. Ces résultats sont-ils si surprenants ou n'étaient-ils pas finalement un peu prévisibles? Crozier ${ }^{16}$ a écrit qu' «on ne change pas la société par décret», que nos modèles sont dépassés et «tendent en outre à se dégrader». Il ajoute que "pour pouvoir régulariser la société, il faut moins de règles... pour pouvoir la gouverner, il faut moins d'autorité formelle». Les spécialistes de l'organisation ne s'y trompent guère quand ils écrivent que «l'approche exclusivement analytique poussée à l'extrême mène à une philosophie abstraite et inhumaine» ${ }^{17}$.

16. Crozier, M. (1979), On ne change pas la société par décret, Paris, Grasset.

17. Peters, T., R. Waterman (1983), le Prix de l'excellence, Paris, Inter Éditions. 
Et pourtant, l'universitaire et le haut-fonctionnaire continuent de défendre des principes et de présenter des théories, en tablant sur une approche très formelle et désincarnée. Le savoir théorique ainsi présenté a peu d'emprise sur les réalités et risque fort de mourir d'inanition. Les résultats sont prévisibles : on va d'échec en échec. Ce que Lipton, Martinson et Wilks ${ }^{18}$ ont écrit au sujet du traitement pourrait facilement être généralisé à l'ensemble de nos principes et théories: "Nothing works». Sommes-nous si mauvais ou serait-ce le passage de la théorie à la réalité qui est si difficile à réaliser? L'échec de la criminologie est-il autre chose qu'un échec d'organisation? Cette limite du formalisme jette un nouvel éclairage sur un important cheval de bataille de la criminologie, l'avènement des droits des délinquants, et plus particulièrement des détenus. Quel est le véritable impact de la reconnaissance de ces droits puisque au-delà de ce formalisme, il y a toute une vie réelle et informelle que les droits peuvent difficilement contrôler. Il est fort probable que l'avènement de tels droits règle moins de problèmes qu'on pouvait l'espérer au départ. D'ailleurs, l'intérêt que manifestent depuis quelques temps les administrateurs pénitentiaires dans la reconnaissance de ces droits peut nous indiquer que certaines politiques pénales ont moins d'importance qu'on le dit et contribuent avant tout à rassurer, se protéger et se donner bonne conscience.

En second lieu, les limites du savoir théorique et la complexité des organisations modernes appellent le développement d'un savoir pratique où, pour reprendre l'expression de Crozier $^{19}$, les gens "n'ont pas besoin de théories sur la société et d'idéologies, mais de moyens plus pratiques pour raisonner sur ce qu'ils font... d'une meilleure connaissance des limites et des contraintes de l'action humaine». Ce thème permet à l'auteur d'établir les nouvelles responsabilités des intellectuels : «Certes, il y aura toujours place pour la retraite dans la tour d'ivoire, les fulgurances du génie solitaire et les snobismes... mais une société complexe a besoin d'autres intellectuels, de plus en plus nombreux, pour cuvrer directement sur la réalité.» Son verdict est impitoyable : «L'intellectuel aristocrate est dépassé, déclassé, contesté.»

18. Lipton, D., R. Martinson, J. Wilks (1975, Effectiveness of Correctional Treatment; a Survey of Treatment Evaluation Studies, Springfield M.A., Preager.

19. On ne change pas la société par décret, Paris, Grasset. 
Ce courant de pensée (et d'action) a des appuis dans des secteurs très diversifiés, depuis les autogestionnaires jusqu'aux chefs d'entreprises, Rosanvallon ${ }^{20}$ ne parle-t-il pas de l'autogestion comme d'une "société d'expérimentation» à partir d'initiatives locales? Peters et Waterman ${ }^{21}$, dans leur analyse des meilleures entreprises, nous disent que... «de temps en temps, les individus et les entreprises ont besoin d'agir avant de réfléchir». Ils ajoutent que la première tâche des gestionnaires est «de faire un choix, après coup, parmi les expériences en cours». Dans ces entreprises, les termes à la mode sont "expérimentation, risques, improvisations, détours, confusion, hasard...» et on se méfie des planifications approfondies.

La criminologie a jusqu'ici séparé le savoir et la pratique, limitant le premier à des connaissances abstraites et la seconde à des techniques et recettes. Le véritable défi du savoir pratique, c'est d'opérer une synthèse de deux domaines qui jusqu'ici ont largement fonctionné de façon parallèle.

En troisième lieu, l'essor de l'organisation nous mène aux questions de contrôle. Si les organisations sont «des groupements humains délibérément construits pour poursuivre des buts spécifiques»"2, le contrôle est nécessaire pour s'assurer que les buts sont atteints. Je ne connais pas d'organisation qui ne soit pas préoccupée par le contrôle. Dans certaines, les contrôles sont plus formels, plus évidents; dans d'autres, ils sont plus subtils. Certains organismes communautaires et novateurs semblent souvent n'exercer aucun contrôle : ce n'est qu'apparence car le groupe peut être plus tyrannique qu'un patron et exercer de fortes pressions pour amener les récalcitrants à se conformer. Ailleurs, les patrons qui ont le plus d'autorité sont ceux qui l'utilisent rarement. Les subtilités du contrôle n'en amoindrissent pas son importance.

Le problème du contrôle est un problème majeur dans les organisations et il est grand temps que les criminologues se

20. Rosanvallon, P. (1976), l'Âge de l'autogestion, Paris, Éditions du Seuil.

21. Le Prix de l'excellence, Paris, Inter Éditions. Duculot.

22. Etzioni, A (1971), les Organisations modernes, Gembloux, Duculot. 
déculpabilisent parce qu'ils doivent en exercer sur les délinquants. La recherche d'un équilibre entre la liberté et le contrôle est non seulement un problème d'organisation mais un problème de société. Le problème avec les délinquants n'est pas d'exercer des contrôles mais de n'exercer que des contrôles. C'est pourquoi le dilemme aide-contrôle m'a toujours paru un faux problème. Le professeur d'université qui parle de cette incompatibilité témoigne souvent du contraire par son enseignement, sa pédagogie et ses examens. Ce sont certaines formes de contrôle, notamment celles de nature coercitive, qui rendent une relation d'aide difficile sinon impossible. Le criminologue bénéficie souvent à ce niveau d'une marge de manœuvre plus grande qu'il ne veut bien l'admettre et je pense qu'il aurait intérêt à tirer des leçons du fonctionnement des organisations dans cette recherche d'équilibre entre la liberté et le contrôle.

Cette réflexion sur l'organisation et le contrôle pourrait peut-être nous permettre de replacer dans une plus juste perspective cette idée de l'extension du contrôle social. Plus la criminologie va s'organiser et plus il y aura de pratiques criminologiques organisées, plus il y aura d'occasions de contrôle. À partir de ce qui a été écrit dans les paragraphes précédents, cela va de soi. Mais on pourrait ajouter que plus il y aura de pratiques criminologiques, plus il y aura d'occasions de mal s'organiser et d'établir des contrôles inutiles, donc d'étendre le contrôle.

J'en veux pour exemple le tamisage informel, plutôt «inorganisé», réalisé par la police avec les mineurs délinquants. Il était réel et, on le voit maintenant, plutôt efficace. La méfiance naturelle des intellectuels et des technocrates vis-à-vis les policiers a amené les premiers à penser qu'ils pouvaient faire mieux que ces derniers par le biais de la déjudiciarisation. On connaît le résultat. Des policiers, sans formation spécifique à cet effet, sans appareil administratif, et avec un certain pouvoir discrétionnaire, faisaient tout aussi bien l'affaire. On pourrait alors reprendre la conclusion de Trépanier ${ }^{23}$, citée plus haut, et la généraliser de la façon suivante : il semble bien que l'extension du contrôle social soit un problème dont il faille accepter l'existence à partir du moment où on formalise des programmes. C'est avant tout un problème d'orga-

23. *Mineurs en danger et mineurs délinquants : deux régimes en transformation au Québec et au Canada», Revue de droit pénal et de criminologie, novembre 1984, p. 817-848. 
nisation, surtout formelle. Il peut donc être souhaitable de laisser des mécanismes informels, en apparence désordonnés et peu spécifiques, fonctionner et obtenir des résultats que des infrastructures imposantes pourraient au mieux égaler. Doob ${ }^{24}$ avait déjà prévu le coup lorsqu'il écrivait, à propos des lois sur les jeunes délinquants, que les meilleures actions des gouvernements sont celles qu'ils ne posent pas!

Le contrôle est donc au cœur de l'action organisée : nous n'y voyons rien de répréhensible et c'est même souvent opportun. Il faut toutefois se rappeler que ça peut également être inutile, voire même déplacé.

En dernier lieu, une réflexion sur les relations entre l'organisation et la criminologie ne peut être complète sans parler du pouvoir. La phrase de Galbraith, citée en exergue au début du texte, indique bien que le pouvoir c'est l'essence de l'action organisée. Galbraith ${ }^{25}$ emprunte à Weber une définition communément admise du pouvoir : «la possibilité de marquer de sa volonté le comportement d'autres personne». "C'est vrai du professeur avec l'élève, du criminologue avec le délinquant, du patron avec l'employé, mais aussi de l'employé avec le patron». Le pouvoir, c'est ce par quoi on existe dans une organisation. Quand on veut faire valoir des idées ou tout simplement utiliser son expertise, il faut posséder du pouvoir sinon nos idées demeurent de vagues souhaits et notre expertise un talent inutile. Il n'y a rien de plus concret dans l'organisation que le pouvoir, même si ce n'est pas quantifiable.

Comme on l'a noté pour le contrôle, le pouvoir et les jeux de pouvoir ne sont pas toujours évidents mais ils sont toujours réels et souvent subtils. La criminologie s'est jusqu'à maintenant plutôt limitée à dénoncer le pouvoir et ses abus. Les criminologues sont souvent gênés d'en parler, se contentant de déplorer qu'ils en possèdent peu. Pourtant, une relation d'aide est-elle autre chose qu'une relation de pouvoir? L'intervention est-elle autre chose qu'un jeu de pouvoir? Il n'y a pas à s'en étonner, encore moins à s'en offusquer - c'est un constat de réalité. «Le pouvoir peut être

24. *Montée et déclin de la déjucidiarisation dans la législation sur les mineurs délinquants au Canada», Déviance et société, vol. 4, no 3 , p. 231-243.

25. Anatomie du pouvoir, Paris, Éditions du Seuil. 
socialement mauvais; il est aussi socialement essentiel» ${ }^{26}$. S'il faut se méfier des abus, il ne faut pas oublier les nécessités. Comme c'est souvent le cas, le mot rebute plus que la réalité. Le chercheur qui, du haut de sa tour d'ivoire, veut faire progresser l'état des connaissances et ainsi influencer l'action sociale est-il si différent de l'intervenant qui veut réduire la violence dans la société.

En dernière analyse, on n'a guère le choix : ou on se préoccupe du sexe des anges ou on essaie d'influencer le cours des choses. Le chercheur qui ne se débarrasse pas de ses cellères idéologiques fait un usage de pouvoir aussi important que le criminologue qui suspend une libération conditionnelle. Malgré tout, la recherche demeure aussi nécessaire que le contrôle des comportements inacceptables.

Cette réflexion sur la pertinence du pouvoir nous permet de replacer dans sa juste perspective la notion de pouvoir discrétionnaire, plutôt discréditée jusqu'à maintenant. Disons tout d'abord que, dans tout pouvoir, il y a une marge de jeu laissée au décideur et, dece fait, tout pouvcir est discrétionnaire. Ensuite, le pouvoir discrétionnaire n'est pas nécessairement mauvais : par exemple, comme nous l'avons vu dans l'étude de la déjudiciarisation, le pouvoir discrétionnaire des policiers était très probablement de beaucoup préférable aux normes bureaucratiques des professionnels. Ceci nous rappelle que l'application de normes bureaucratiques, théoriquement sans discrétion, constitue certes un des gestes les plus tyranniques de notre société. Le pouvoir discrétionnaire, fondé sur le jugement, est certes préférable à la rigidité bureaucratique ou légale. Un jugement est contestable; une norme ou une loi est inattaquable, si ce n'est au prix de délais juridiques longs et coûteux. Vouloir éliminer le pouvoir discrétionnaire, c'est vouloir éliminer les relations humaines et les remplacer par des rites momifiés et désincarnés. Je préfère l'excès de vie à l'absence de vie. Celui ou celle qui connaît l'action sociale sait ce dont je parle. Les seuls criminologues dont l'action a encore un sens sont ceux qui ont refusé de joindre le train de la bureaucratie, des lois et des normes, et qui misent encore sur les relations humaines, si imparfaites soient-elles.

Finalement, on ne peut y échapper : organisation, pouvoir et contrôle forment un tout indissociable, car «l'organisation cons- 
titue la source dernière de tout pouvoir ${ }^{27}$. Au-delà de l'angélisme et des fausses craintes existentielles, la criminologie doit y étendre son savoir si elle ne veut pas perdre «sa signification et sa pertinence». 